Tópico de Interesse Geral

\title{
Intoxicação por Senecio spp. em bovinos no Rio Grande do Sul: condições ambientais favoráveis e medidas de controle ${ }^{1}$
}

\author{
Fernando Castilhos Karam ${ }^{2 *}$, Ana Lucia Schild ${ }^{3}$ e João Roberto Braga de Mello ${ }^{4}$
}

\begin{abstract}
Karam F.C., Schild A.L. \& Mello J.R.B. 2011. [Poisoning by Senecio spp. in cattle in southern Brazil: Favorable conditions and control measures.] Intoxicação por Senecio spp. em bovinos no Rio Grande do Sul: condições ambientais favoráveis e medidas de controle. Pesquisa Veterinária Brasileira 31(7):603-609. Instituto de Pesquisas Veterinárias Desidério Finamor, Fundação Estadual de Pesquisa Agropecuária, Estrada do Conde 6000, Eldorado do Sul, RS 92990-000, Brazil. E-mail: fernandockaram@yahoo.com.br

The study aimed to review the main aspects of Senecio spp. poisoning in Rio Grande do Sul, sou-thern Brazil, in relation to the pathology, pathogenesis and epidemiology of this important cause of death in cattle in that State. The main climatic and environmental factors that apparently favor the emersion and plant establishment were revised. The occurrence of poisoning, which has increased its frequency in recent years in the state, and possible ways to control the plant, including the correct handling of soil and the use of less susceptible domestic species in invaded areas were also discussed.
\end{abstract}

INDEX TERMS: Pyrrolizidine alkaloids, seneciosis, poisonous plants, environment, cattle, plant poisoning.

RESUMO.- Este trabalho teve por objetivo revisar os principais aspectos da intoxicação por Senecio spp. no Rio Grande do Sul no que se refere à patologia, patogenia e epidemiologia dessa importante causa de morte em bovinos nesse Estado. Foram abordados, também, os principais fatores climáticos e ambientais que aparentemente favorecem a emergência e o estabelecimento da planta e a ocorrência da intoxicação, que tem aumentado a sua frequência nos últimos anos no Estado, e as possíveis formas de controle da planta incluindo o manejo correto do solo e a utilização de espécies domésticas menos susceptíveis nas áreas invadidas.

TERMOS DE INDEXAÇÃO: Alcaloides pirrolizidínicos, seneciose, plantas tóxicas, ambiente, bovinos, intoxicação por planta.

\section{INTRODUÇÃO}

A intoxicação por Senecio spp. (seneciose) é a mais frequente das intoxicações atribuídas a plantas e uma das principais

\footnotetext{
${ }^{1}$ Recebido em 8 de fevereiro de 2011.

Aceito para publicação em 22 de março de 2011.

${ }^{2}$ Instituto de Pesquisas Veterinárias Desidério Finamor (IPVDF), Fepagro, Estrada do Conde 6000, Eldorado do Sul, RS 92990-000, Brasil. *Autor para correspondência: fernandockaram@yahoo.com.br

${ }^{3}$ Laboratório Regional de Diagnóstico, Faculdade de Veterinária, Universidade Federal de Pelotas (UFPel), Campus Universitário s/n, Pelotas, RS 96010-900, Brasil.

${ }^{4}$ Departamento de Farmacologia, Instituto de Ciências Biológicas (ICBS), Universidade Federal do Rio Grande do Sul (UFRGS), Rua Sarmento Leite 500, sala 202, Porto Alegre, RS 90050-170, Brasil.
}

causas de morte entre bovinos no Rio Grande do Sul (RS) (Barros et al. 2007, Grecco et al. 2010, Lucena et al. 2010a,b, Karam \& Motta 2011). Estima-se que no mínimo 5\% da população bovina morrem anualmente (Méndez \& Riet-Correa 2008) e dados de laboratórios de diagnóstico mostram que 10,6-14\% desses casos devem-se à intoxicação por plantas (Riet-Correa et al. 2007). No RS, com uma população bovina de $13 \mathrm{mi}-$ lhões de cabeças, as mortes por diferentes causas representam 650.000 bovinos por ano e pode-se estimar que as perdas anuais em decorrência da ingestão de plantas tóxicas variam de 68.900 a 91.000 bovinos. Metade dessas mortes é causada por diferentes espécies de Senecio e considerando um preço médio de US\$ 200 por animal, as perdas diretas atribuídas à seneciose no RS são de aproximadamente US\$ 7,5 milhões por ano (Méndez \& Riet-Correa 2008).

A ocorrência dessa toxicose, sua patogenia e sinais clínicos têm sido amplamente estudados (Tokarnia et al. 2000, Basile et al. 2005, Pedroso et al. 2007, Riet-Correa et al. 2007, Rissi et al. 2007, Méndez \& Riet-Correa 2008, Santos et al. 2008, Grecco et al. 2010, Lucena et al. 2010a,b). No entanto, a frequência da seneciose e a ineficácia de medidas terapêuticas ratificam sua importância e têm justificado novos estudos a respeito. Este artigo inclui um breve histórico do gênero Senecio, a seneciose e as condições ambientais favoráveis à intoxicação no RS e trata das medidas para o controle da intoxicação.

\section{BREVE HISTÓRICO DO GÊNERO SENECIO}

0 gênero Senecio pertence à família Asteraceae e é cosmopolita, mas a maior distribuição do gênero é na América do 
Sul com cerca de 500 espécies. É o gênero mais importante da tribo Senecioneae e o maior dentre as fanerógamas (que possuem flores), com cerca de 3.000 espécies distribuídas em todo o mundo (Matzenbacher 1998). Somente não ocorrem Senecio spp. nas regiões polares e na Amazônia e, no Brasil, existem 85 espécies (Cabrera \& Klein 1975). Segundo Motidome \& Ferreira (1966), há 128 espécies deste gênero só no Brasil, embora algumas delas sejam pouco comuns.

No RS a ocorrência de Senecio foi registrada entre 18201821 na região do município de Rio Grande (Saint-Hilaire 1887). Matzenbacher $(1998,2009)$ constatou a existência de 26 espécies, três variedades, uma forma ${ }^{5}$ e um híbrido do gênero Senecio, no Estado.

\section{SENECIOSE}

A intoxicação por Senecio spp., especialmente por Senecio brasiliensis, comum do estado de São Paulo ao sul do Brasil (Tokarnia et al. 2000, Riet-Correa et al. 2007), é considerada das mais importantes nessas regiões e, juntamente com outras espécies do gênero, a causa de expressivo prejuízo na bovinocultura do RS (Rissi et al. 2007).

No RS, dentre as diversas espécies do gênero Senecio, $S$. brasiliensis, S. oxyphyllus, S. heterotrichius e S. selloi, são frequentemente associadas com casos de intoxicação em bovinos (Méndez \& Riet-Correa 2008). Há, também, registro da intoxicação por S. tweediei (Méndez \& Riet-Correa 1993) e mais recentemente, também no RS, por $S$. madagascariensis, que está em franca expansão pelo Estado com registro de casos de intoxicação em função dessa expansão (Matzenbacher \& Schneider 2008, Cruz et al. 2010, Karam et al. 2011).

A intoxicação por Senecio acomete principalmente bovinos (Barros et al. 1987, Mendéz et al. 1987, Driemeier et al. 1991, Barros et al. 1992), mas podem ocorrer surtos, embora com menor frequência, em equinos (Carvalho \& Maugé 1946, Curial \& Guimarães 1958, Gava \& Barros 1997), ovinos (Ilha et al. 2001, Grecco et al. 2011b), caprinos e suínos (Dollahite 1972 e Forsyth 1979 apud Tokarnia et al. 2000) e búfalos (Corrêa et al. 2008). Experimentalmente S. brasiliensis também é tóxico para aves (Méndez et al. 1990).

Das espécies de Senecio descritas no mundo, aproximadamente 25 têm sido comprovadas como tóxicas para animais domésticos ou para o homem. Tokarnia et al. (2000) mencionam que doenças enzoóticas caracterizadas por lesão hepática crônica e descritas anteriormente sob outras denominações, tratavam-se de intoxicação por uma ou mais espécies de Senecio.

As espécies tóxicas contêm alcaloides pirrolizidínicos (APs) que são primariamente metabolizados pelos hepatócitos através do sistema citocromo p-450, em grupos pirrois responsáveis pelo dano hepatocelular (Spinosa 2008, Cullen 2009). A toxicidade varia em função dos metabólitos formados e da espécie animal ter capacidade de detoxificá-los ou não (Maxie 2007). Os metabólitos ligam-se ao ácido desoxirribonucleico (DNA), inibindo a mitose dos hepatócitos, cujo núcleo segue sintetizando DNA, resultando num au-

\footnotetext{
${ }^{5}$ Em botânica é uma categoria definida por quem descreve e estabelece categoria inferior à variedade, de origem genética, geralmente relacionada a uma característica mais sutil como cor e matiz.
}

mento de tamanho da célula (Osweiler 1998). Apesar de o fígado ser o órgão mais atingido, parte dos pirrois sintetizados atinge a circulação geral e pode causar nefrose e pneumonia intersticial uma vez que as enzimas do citocromo p-450 estão presentes e, portanto, metabolizam APs em pirrois diretamente nesses órgãos (Santos et al. 2008, Cullen 2009). As células lesadas têm seu metabolismo diminuído evoluindo até a morte de forma progressiva e irreversível. Os animais manifestam os sinais clínicos de forma variada, de acordo com o grau de comprometimento orgânico e variações biológicas individuais (Radostits et al. 2002), que podem ser divididas em fatores internos (espécie animal, fatores genéticos, sexo, idade, prenhez e presença de doenças) e externos (dieta e ambiente) (Spinosa et al. 2008).

A lesão hepática produzida pelos APs normalmente causa a morte do animal. Essa lesão pode determinar edema da substância branca (status spongiosus) no sistema nervoso central (SNC) pela hiperamonemia que ocorre devido a síntese inadequada de uréia no fígado. No SNC, a amônia é metabolizada por astrócitos, eventualmente convertida em glutamina, que tem ação neurotóxica, determinando o edema (Hooper 1972, Barros et al. 1992, Summers et al. 1995, Radostits et al. 2002, Rissi et al. 2010). 0 quadro clínico mais característico é de encefalopatia hepática com apatia ou hiperexcitabilidade, incoordenação, agressividade, tenesmo, diarréia e, ocasionalmente, prolapso retal, com um curso clínico, geralmente, de 24-96 horas. Alguns animais apresentam emagrecimento progressivo, com diarréia ou não, por um período de até três meses, podendo manifestar, antes da morte, encefalopatia hepática, ou permanecerem em decúbito até a morte. Intoxicação por APs pode cursar, também, com fotossensibilização e, neste caso, o curso clínico costuma ser mais prolongado, geralmente entre 30 e 60 dias (Riet-Correa \& Méndez 2007).

Estudos recentes comprovam interferência no desenvolvimento físico e neurocomportamental na prole de ratas expostas ao extrato de $S$. brasiliensis no período pré-natal (Dalmolin et al. 2010, Sandini et al. 2010) e, talvez, futuramente, essa interferência deva ser considerada em animais de produção, incluindo os bovinos. Devido à ação cancerígena e mutagênica dos APs, há que considerar, também, os riscos potenciais à saúde humana (Méndez \& Riet- Correa 2008, Spinosa et al. 2008).

Testes de função hepática (indicativos da intoxicação), assim como os estudos histológicos de biopsias hepáticas podem ser eficientes para estabelecer um prognóstico naqueles animais não afetados clinicamente. 0 diagnóstico por biopsia hepática é um meio rápido e seguro para detectar se os animais estão afetados ou não e permitir o abate antes que perdas econômicas maiores ocorram (Barros et al. 2007). No entanto, a intoxicação em humanos pela ingestão de carne de animais intoxicados por plantas, no Brasil, até o momento, não é conhecida, e sabe-se que os APs são metabolizados no fígado em metabólitos pirrólicos e resíduos desses metabólitos podem ser encontrados em diversos tecidos do animal durante longos períodos após cessar a ingestão dos alcaloides (Méndez \& Riet-Correa 2008).

Em bovinos, a morbidade da intoxicação por Senecio spp. é variável entre $1 \%$ e $30 \%$ e a letalidade é praticamente $100 \%$. 
O impacto econômico se dá pelas perdas diretas por morte, falhas na reprodução, baixa produtividade pela manifestação subclínica da doença, além de uma maior suscetibilidade para outras doenças devido à depressão imunológica dos animais. As perdas econômicas indiretas incluem o custo do controle das plantas nas pastagens, a desvalorização dessas pastagens, medidas de manejo alternativas, gastos com a reposição dos animais perdidos e os custos relacionados com o diagnóstico e tratamento dos animais afetados (Méndez \& Riet-Correa 2008).

\section{CONDIÇÕES FAVORÁVEIS À INTOXICAÇÃO NO RS (CLIMA, SOLO, MANEJO)}

No RS o maior risco de ingestão de Senecio spp. ocorre pelo pastoreio direto, em épocas de pouca oferta de pasto, uma vez que a planta é pouco palatável e consumida pelos bovinos somente sob determinadas condições (Méndez \& RietCorrea 2008, Spinosa et al. 2008). A superlotação de bovinos e a grande quantidade de Senecio spp. favorecem a ingestão e, se as plantas novas estão estreitamente associadas ao capim, o perigo de ingestão pelos bovinos é ainda maior (Tokarnia \& Döbereiner 1984, Schild et al. 1989, Driemeier et al. 1991, Driemeier \& Barros 1992, Tokarnia et al. 2000).

Em um surto diagnosticado em búfalos em Nova Prata (RS), durante o inverno de 2006, os fatores epidemiológicos associados foram a alta infestação de $S$. brasiliensis no campo e a forte estiagem (Corrêa et al. 2008). A ingestão pode, também, ser acidental através do feno, silagem ou grãos contaminados (Peterson \& Culvenor 1983, Coombs et al. 1997, Radostits et al. 2002). Fenos ou silagens contaminados por plantas que contém APs podem resultar em intoxicação dos animais que os consumirem apesar de que na silagem há perda de $20 \%$ a $30 \%$ no conteúdo dos alcaloides (Méndez 1993). Em Santa Catarina, em um surto em bovinos leiteiros, foi relatado que os animais recebiam feno de alfafa à vontade, cujos fardos apresentavam infestação média de 3\% de Senecio spp. e, no Paraná, um surto em bovinos mestiços foi devido a grande infestação do campo nativo com S. brasiliensis, sem deficiência de pastagem (Basile et al. 2005).

Toda a espécie tóxica de Senecio contém APs distribuídos por toda a planta. 0 teor de alcaloides é variável entre as espécies e, provavelmente, de ano para ano, entre regiões e condições ambientais diferentes (Kingsbury 1964, Hirschmann et al. 1987, Habermehl et al. 1988, Méndez et al. 1990, Méndez 1993, Tokarnia et al. 2000). Na espécie madagascariensis o conteúdo de APs é maior nas flores, especialmente durante a primavera, assim como no conjunto das partes aéreas (Karam et al. 2011). A variedade de alcaloides presentes e a variação no teor de cada um podem interferir diretamente no efeito tóxico do agente (MacLachlan \& Cullen 1998) e, consequentemente, na manifestação da doença.

A manifestação clínica da doença pode ser observada em todas as épocas do ano existindo alguma variação entre as diferentes regiões do Estado, provavelmente devido às diferenças ambientais (Grecco et al. 2010, Karam \& Motta 2011). Uma vez que a lesão desenvolve-se lentamente (Bull 1955) é provável que os animais ingiram a planta em estações de carência de forragem e, no caso de surtos na primavera, as es- tações anteriores (outono-inverno), coincidem com a época de maior emergência de Senecio spp. e de maior concentração de APs nas espécies estudadas no RS (Karam et al. 2004). Condições de maior demanda fisiológica por situações estressantes, comuns na primavera, como parição, feiras e transporte, podem desencadear a intoxicação latente (Dickinson 1980, Johnson \& Smart 1983), já que na ausência de maior exigência do organismo, o dano hepático pode não se manifestar (Peterson \& Culvenor 1983).

Em propriedades com casos de intoxicação observa-se, em geral, infestação de Senecio spp. em diferentes estágios vegetativos e com bom vigor e sempre pouca oferta de pasto em relação ao número de animais no estabelecimento (Karam et al. 2004).

O ciclo de vida e o comportamento de plantas estão diretamente ligados aos fatores ambientais (especialmente precipitação, fotoperíodo, temperatura do ar e do solo), pois regulam os fenômenos biológicos (Borgignon \& Piccolo 1981, Beskow 1995, Madanes et al. 1996). No RS, S. brasiliensis é considerada uma espécie perene enquanto $S$. oxyphyllus, $S$. heterotrichius e $S$. selloi são anuais (Matzenbacher 1998). Essas espécies comportam-se como anuais e monocárpicas, com algumas variações individuais (Karam et al. 2002). Conforme os danos sofridos, como pisoteio excessivo, corte etc., as plantas podem comportar-se como anuais, bianuais, ou até mesmo perenes. Se os danos forem intensos e/ou frequentes, uma porção de plantas apresentará um ciclo bianual, com a maioria precisando de dois ou mais anos para florescer. Se as condições de crescimento são sempre favoráveis, algumas plantas podem florescer no primeiro ano, comportando-se como anuais (Beskow 1995). S. madagascariensis comporta-se como anual e pluricárpica (Karam 2010, dados não publicados). Portanto, se as condições ambientais são favoráveis à emergência e ao desenvolvimento, pode haver disponibilidade da planta em qualquer época do ano e, consequentemente, a ingestão e a intoxicação em diferentes épocas.

Fatores ambientais como frio excessivo no inverno ou déficit hídrico no verão e formas inadequadas de manejo como fogo, carga animal excessiva, entre outros, determinam as condições das pastagens naturais (Crawshaw et al. 2007, Overbeck et al. 2007). Em um estudo na região Sudoeste do $\mathrm{RS}$, esses fatores favoreceram o aparecimento de manchas de solo desnudo entre a vegetação remanescente e o estabelecimento de plantas indesejáveis (Gonçalves \& Girardi-Deiro 1986, Girardi-Deiro et al. 1992). Um pastoreio pesado no inverno, ou em época de alta precipitação, contribui para a diminuição da camada de cobertura vegetal, aumentando a incidência de luz no solo e, consequentemente, a temperatura, o que favorece a germinação das sementes ali presentes. Em fanerógamas, a germinação de sementes responde às flutuações diurnas de temperatura e essa resposta varia de acordo com a amplitude dessa flutuação e a presença ou ausência de luz (Thompson et al. 1977, Coombs et al. 1991, Beskow 1995). No RS, a grande amplitude térmica pode ser um dos fatores que favorece a ocorrência de Senecio spp. (Karam \& Jarenkow 2011). Há correlação positiva entre a vegetação de cobertura, principalmente para espécies perenes, e o banco de sementes no solo (BSS), indicando que o BSS pode possuir importante papel na dinâmica da vegetação natural campestre 
(Maia et al. 2003, 2004). Plantas consideradas daninhas podem ser disseminadas via seminífera ou vegetativa, especialmente nas espécies perenes, muitas vezes por ação do homem. Do ponto de vista morfofisiológico a fixação de plantas envolve complexos aspectos morfogênicos e edafoclimáticos. Condições ambientais favoráveis, como adequado suprimento hídrico, temperatura, concentração de oxigênio e presença ou ausência de luz, conforme ela seja fotoblástica positiva ou negativa, determinam o processo de germinação (Silva \& Silva 2009).

Na espécie $S$. jacobaea a emergência de mudas é mais alta no solo descoberto do que em locais com vegetação de cobertura densa e é maior no solo úmido, no inverno, quando a temperatura está baixa. A germinação de sementes é impedida no verão pela falta de umidade e em qualquer época do ano pela presença da cobertura de pastagem. Não há influência direta, pelo pisoteio, à emergência de mudas. 0 pisoteio geralmente estimula a germinação através do dano que causa à vegetação de cobertura. 0 pisoteio é determinante para formação de solo nu no inverno, quando as plantas são mais severamente danificadas. A emergência de S. jacobaea foi mínima onde a pastagem de cobertura não foi perturbada. A presença de $S$. jacobaea e $S$. aquaticus aumenta à medida que diminui a cobertura da vegetação e, além disso, solos mais ricos em fósforo têm menor infestação de $S$. jacobaea. Um baixo $\mathrm{pH}$ do solo parece favorecer o seu aparecimento (Beskow 1995, McClements et al. 1998). Baixo pH e baixo teor de fósforo são fatores comuns em solos de várias regiões do RS (Macedo 1984). Portanto, esses fatores devem ser considerados, também, na ocorrência de Senecio spp. no Estado.

A ocorrência da seneciose é maior do que o número de registros em laboratórios de diagnóstico (Grecco et al. 2010, Karam \& Motta 2011) e o crescente aumento de casos em bovinos no RS pode ser atribuído, em parte, ao declínio acentuado da ovinocultura no Estado e, consequentemente, ao aumento da população da planta (Karam et al. 2004), semelhante ao que ocorreu na Grã-Bretanha nos anos de 1940 (Harper $\&$ Wood 1957). Alguns autores sugerem que o aumento da ocorrência em bovinos e a mudança no padrão da intoxicação, com apresentação subaguda da doença, podem estar relacionados às mudanças climáticas (Grecco et al. 2011a). Embora os ovinos possam adoecer espontaneamente (Ilha et al. 2001, Grecco et al. 2011b), a intoxicação não é comum nessa espécie por ser mais resistente à ação dos alcaloides e, por isso, podem ser usados como controladores naturais da planta. No RS, nas áreas onde há ovinos em pastoreio, geralmente não há Senecio spp. (Méndez \& Riet-Correa 2008). Deve considerar-se, porém, pelo menos no caso da espécie $S$. jacobaea, que a dispersão de sementes pode ocorrer pelo estrume de ovinos que se alimentaram com a planta na frutificação, cujas sementes não são danificadas no trato digestivo e podem germinar (Harper \& Wood 1957).

\section{MEDIDAS DE CONTROLE}

Não existe tratamento específico nem sintomático que permita recuperar os animais com sinais clínicos da doença (RietCorrea et al. 2007). Há, experimentalmente, tentativas de controle da intoxicação, alterando-se o processo de biotransformação hepática dos APs. Para tanto, são utilizados aminoáci- dos de forma a suprir os radicais sulfidrila para a conjugação com os pirrois, como por exemplo, a cisteína (Spinosa et al. 2008).

Deve-se levar em conta que a maioria das intoxicações por plantas acontece em animais que, pelo menos em algum período do ano ou até no ano anterior, passaram por um período de restrição alimentar. No RS essa situação é comum de ocorrer no outono e inverno quando a disponibilidade de forragem diminui consideravelmente (Méndez \& Riet-Correa 2008), especialmente em regiões onde o frio é mais rigoroso e também por excesso de chuvas ou em casos de seca. Excesso de plantas indesejáveis, normalmente em campos muito alterados, decorrentes de um desequilíbrio na biocenose (associação entre a macro, meso e micro vida de uma área, especialmente a alimentar) (Romero 1998), dificulta a seleção no pastoreio. Deve ser considerado, ainda, que os bovinos não são hábeis em fazer essa seleção. Portanto, manter uma adequada oferta de pasto de boa qualidade em relação à lotação animal, especialmente nas épocas críticas do ano no sul do RS é fundamental para evitar a ingestão de Senecio no período em que as espécies têm maior teor de APs (Karam et al. 2004). Nas áreas mais invadidas pela planta devem ser colocadas as categorias que irão permanecer menor tempo no estabelecimento ou fazer rodízio das diferentes categorias nos diferentes campos (Méndez \& Riet-Correa 2008).

Para controle da população da planta devem ser evitadas práticas que resultem em diminuição da cobertura vegetal e a permanência de solo desprovido de vegetação, especialmente importantes para a germinação de sementes e o estabelecimento de plantas fotoblásticas positivas, como Senecio spp. (Karam \& Jarenkow 2011).

Arrancar a planta com raiz em dias de solo úmido antes da floração, que no RS se concentra de setembro a dezembro para a maioria das espécies, é bastante eficaz, porém não é uma medida prática para grandes extensões e, ainda, pode haver rebrote dos restos de raízes que ficam no solo. Essa medida não é recomendada por Beskow (1995) pela capacidade de propagação vegetativa (Silva \& Silva 2009), no entanto, tem sido recomendada para as espécies em que a roçada é ineficiente, como S. madagascariensis (Amaro 2005).

Roçadas podem ser feitas, também, antes da floração, sempre evitando a produção e dispersão de sementes. Essa prática deve ser repetida quando os rebrotes atingem 10-15 cm de altura, a fim de esgotar as reservas nutritivas da planta até seu desaparecimento (Amaro 2005). Essas roçadas podem ser associadas ao pastoreio com ovinos. Esse método tem sido adotado com sucesso por alguns produtores rurais que mencionam que na passagem da fase vegetativa para reprodutiva de Senecio spp., pouco antes da floração, o campo foi roçado e isso diminuiu cerca de $90 \%$ o porte da planta. Quando a mesma rebrotou e atingiu a fase adulta, novamente floresceu rente ao chão, foram colocados os cordeiros da propriedade e notou-se que os animais comiam Senecio mesmo quando havia no potreiro ainda bom pasto. Foi observado, também, que num período de três anos de seca houve uma troca na composição botânica dos campos e que dentre as espécies de herbáceas que apareceram uma das principais foi Senecio, porém em campos com ovinos não se encontrava um único exemplar da planta (Kluwe 2008). 
O pastoreio com ovinos pode ser feito conjuntamente com os bovinos, já que aqueles consomem e controlam a planta, no entanto, deve ser considerada a possibilidade de que os ovinos introduzidos em áreas muito infestadas por Senecio spp. possam intoxicar-se (Ilha et al. 2001, Allan et al. 2005, Méndez \& Riet-Correa 2008, Grecco et al. 2011b). Uma lotação de ovinos igual ou maior que 0,43 animais por hectare, em pastoreio contínuo por no mínimo 30 dias, controlou a ocorrência de Senecio spp. (Soares et al. 2000).

A prática de fazer feno ou silagem de áreas invadidas por Senecio spp. deve ser desestimulada. A dessecação da planta reduz seu potencial tóxico, porém impossibilita os bovinos de selecionar as plantas, as quais podem tornar-se, também, mais palatáveis, assim como se tornam mais palatáveis quando são cortadas, arrancadas ou pulverizadas com herbicidas (Beskow 1995).

O controle químico tem sido indicado para espécies que possuem capacidade de propagação vegetativa, mas deve ser feito como método auxiliar dentro de um manejo integrado (Silva \& Silva 2009). Para o controle de S. jacobaea os herbicidas podem ser usados na brotação desde que seja estimulado o crescimento da pastagem ao redor (Beskow 1995). Para a espécie $S$. madagascariensis recomendam-se aplicações localizadas de herbicidas pós-emergentes pouco residuais quando as plantas têm cerca de cinco folhas, de modo que seja eficiente a dose mínima, o que também diminui custos. 0 herbicida não atua sobre as sementes, de modo que esse método deverá ser repetido por alguns anos até o esgotamento do BSS. Tanto o controle mecânico como o químico devem ser feitos no inverno, até 10 de agosto, se as condições climáticas não anteciparem a floração (Amaro 2005). Deve-se considerar que $S$. madagascariensis tem várias florações durante um ano e, especialmente nesta espécie, os controles mecânicos têm sido pouco eficientes. A simultaneidade das fenofases vegetativas e reprodutivas em uma mesma planta e na população de plantas, assim como o curto período entre emergência e floração (6-10 semanas), são determinantes de uma baixa eficiência de um ou outro método e sugerem a necessidade de múltiplas operações de controle (Allan et al. 2005, Villalba \& Fernández 2005).

Alguns países, como Nova Zelândia (NZ), possuem legislação específica sobre prevenção, controle e erradicação de plantas nocivas à saúde e/ou economia, incluindo Senecio spp., e faz parte de ações governamentais regulares, sendo as propriedades passíveis de fiscalização. Baseado em um estudo sobre dispersão de sementes em plantas da família Asteraceae, uma lei na NZ proíbe a existência de $S$. jacobaea na faixa de $20 \mathrm{~m}$ da divisa da propriedade e proíbe que o produtor tenha plantas em flor na sua fazenda (Beskow 1998, MAF 2010). No RS a Associação Rio-Grandense de Empreendimentos de Assistência Técnica e Extensão Rural (Emater), juntamente com a Fundação Estadual de Pesquisa Agropecuária e sua unidade em saúde animal, o Instituto de Pesquisas Veterinárias Desidério Finamor (IPVDF), produziu um folder sobre seneciose dentro do Programa Pecuária Familiar, alertando sobre o problema e indicando algumas medidas de controle. Esse material teve repercussão positiva na cadeia produtiva, o que demonstra a carência e potencialidades de trabalho nessa área (Emater 2008).
Estão em desenvolvimento trabalhos com o uso de ovinos de acordo com a infestação da planta para que se possa recomendar medidas práticas, seguras e eficazes de controle da população de Senecio spp., sempre considerando a importância de um manejo adequado do campo e pastagens para evitar o solo descoberto (Karam 2010), estudos associando o pastoreio com ovinos e bovinos (Cruz 2010) e, também, estudos visando o controle pela utilização de insetos, especialmente de Phaedon confinis em $S$. brasiliensis, a exemplo do que já foi feito com sucesso em outros países (Coombs et al. 1991, 1996, 1997, McEvoy et al. 1991, Mendes et al. 2005, Milléo et al. 2006, Solera et al. 2007).

Diante dos graves prejuízos causados pela seneciose, justifica-se a busca de alternativas de controle dessa importante causa de morte em bovinos no RS. Medidas que aliam controle biológico de Senecio spp. ao manejo correto da terra são menos agressivas ao ambiente natural e se constituem numa forma de redução de prejuízos econômicos a médio prazo.

Agradecimentos.- Ao INCT/CNPq (Proc.nํ573534/2008-0).

\section{REFERÊNCIAS}

Allan H., Launders T. \& Walker K. 2005. Fireweed. Primefact 126:1-8. State of New South Wales://www.dpi.nsw.gov.au/

Amaro C. 2005. Maleza invasora y de cuidado para el pastoreo: el Senecio, p.4-9. In: Lechuza Roja 3(9) set. 2005. Publicación de Laboratorios Santa Elena S.A., Montevideo, Uruguay.

Barros C.S.L., Driemeier D., Pilati C., Barros S.S. \& Castilhos L.M.L. 1992. Senecio spp. poisoning in cattle in southern Brazil. Vet. Hum. Toxicol. 34(3):241-246.

Barros C.S.L., Castilhos L.M.L., Rissi D.R., Kommers G.D. \& Rech R.R. 2007. Biópsia hepática no diagnóstico da intoxicação por Senecio brasiliensis (Asteraceae) em bovinos. Pesq. Vet. Bras. 27(1):53-60.

Basile J.R., Diniz J.M.F., Okano W., Cirio S.M. \& Leite L.C. 2005. Intoxicação por Senecio spp. (Compositae) em bovinos no sul do Brasil. Acta Scient. Vet. 33:57-62.

Beskow W.B. 1995. A study of the factors influencing the emergence and establishment of ragwort (Senecio jacobaea L.) seedlings in pastures. MSc. Dissertation, Massey University, New Zealand. 116p.

Beskow W.B. 1998. Comunicação pessoal (Massey University, New Zealand). Borgignon O.J. \& Piccolo A.L.G. 1981. Fenologia de Hydrocotyle leucocephala Cham. Rodriguésia 33(56):91-99.

Bull L.B. 1955. The histological evidence of liver damage from pyrrolizidine alkaloids: Megalocytosis of the liver cells and inclusion globules. Aust. Vet. J. 31:33-40.

Bull L.B., Culvenor C.C.J. \& Dick A.T. 1968. The pyrrolizidine alkaloids. Their chemistry, pathogenicity and other biological properties. North-Holland Publ. Co., Amsterdam. 293p.

Cabrera A.L. \& Klein R.M. 1975. Compostas. 2. Tribo: Senecioneae, p.126222. In: Reitz R. (Ed.), Flora Ilustrada Catarinense, Itajaí.

Carvalho G.S.T. \& Maugé G.C. 1946. Ação tóxica de Senecio brasiliensis Lessing, fam. Compositae. Revta Fac. Med. Vet. São Paulo 3(3):131-136.

Curial O. \& Guimarães J.P. 1958. Cirrose hepática enzoótica no cavalo. Mem. Inst. Oswaldo Cruz, Rio de J., 56(2):635-644.

Coombs E.M., Radtke H., Isaacson D.L. \& Snyder S.P. 1996. Economic and regional benefits from the biological control of tansy ragwort, Senecio jacobaea, in Oregon. Proc. IX International Symposium on Biological Control of Weeds, University of Cape Town, Stellenbosch, p.489-494.

Coombs E.M., Bedell T.E. \& McEvoy P.B. 1991. Tansy ragwort (Senecio jacobaea): Importance, distribution and control in Oregon, p.419-428. In: James L.F., Evans J.O., Ralphs M.H. \& Child R.D. (Eds), Noxious Range Weeds. Westview Press, San Francisco.

Coombs E., Mallory-Smith L.C., Burrill R.H., Callihan R., Parker \& Radtke H. 
1997. Tansy ragwort, Senecio jacobaea L. Pacific Northwest Extension Publication 175:1-7.

Corrêa A.M.R., Junior P.S.B., Pavarini S.P., Santos A.S., Sonne L., Zlotowski P., Gomes G. \& Driemeier D. 2008. Senecio brasiliensis (Asteraceae) poisoning in Murrah buffaloes in Rio Grande do Sul. Pesq. Vet. Bras. 28(3):187189.

Crawshaw D., Dall'Agnol M., Cordeiro J.L.P. \& Hasenack H. 2007. Caracterização dos campos sul-rio-grandenses: uma perspectiva da ecologia da paisagem. Boletim Gaúcho de Geografia 33:233-252.

Cruz C.E.F. 2010. Comunicação pessoal (Faculdade de Veterinária, Universidade Federal do Rio Grande do Sul, Porto Alegre).

Cruz C.E.F., Karam F.C., Dalto A.C., Pavarini S.P., Bandarra P.M. \& Driemeier D. 2010. Fireweed (Senecio madagascariensis) poisoning in cattle. Pesq. Vet. Bras. 30(1):10-12.

Cullen J.M. 2009. Doenças do fígado e do sistema biliar, p.418-455. In: McGavin M.D. \& Zachary J.F. (Eds), Bases da Patologia em Veterinária. 4a ed. Elsevier, Rio de Janeiro.

Dalmolin D.P., Ricci E.L., Telloli C.S., Spinosa H.S. \& Górniak S.L. 2010. Efeitos da exposição ao Senecio brasiliensis durante a gestação no desenvolvimento físico e reflexológico da prole de ratas. Anais 4a Semana Científica Benjamin Eurico Malucelli, p.112-113. (Resumo)

Dickinson J.O. 1980. Release of pyrrolizidine alkaloids into milk. Proc. West. Pharmacol. Soc. 23:377-379.

Driemeier D., Barros C.S.L. \& Pilati C. 1991. Seneciose em bovinos. Hora Vet. 59:23-30.

Driemeier D. \& Barros C.S.L. 1992. Intoxicação experimental por Senecio oxyphyllus (Compositae) em bovinos. Pesq. Vet. Bras. 12(1/2):33-42.

Emater 2008. Intoxicação por "maria mole", Seneciose. Publicação Técnica do Programa Pecuária Familiar. Emater/Ascar-RS/Fepagro-IPVDF, Porto Alegre, RS. (Folder)

Girardi-Deiro A.M., Gonçalves J.O.N. \& Gonzaga S.S. 1992. Campos naturais ocorrentes nos diferentes tipos de solos no município de Bagé, RS. 2. Fisionomia e composição florística. Iheringia, Sér. Bot., Porto Alegre, 42:55-79.

Gonçalves J.O.N. \& Girardi-Deiro A.M. 1986. Efeito de três cargas animais sobre a vegetação de pastagem natural. Pesq. Agropec. Bras. 21(5):547554.

Grecco F.B., Schild A.L., Estima-Silva P., Marcolongo-Pereira C., Soares M.P. \& Sallis E.S.V. 2010. Aspectos epidemiológicos e padrões de lesões hepáticas em 35 surtos de intoxicação por Senecio spp. em bovinos no sul do Rio Grande do Sul. Pesq. Vet. Bras. 30(5):389-397.

Grecco F.B., Marcolongo-Pereira C., Soares M.P., Sallis E.S.V. \& Schild A.L. 2011a. Possible association between precipitation and incidence of $\mathrm{Se}$ necio spp. poisoning in cattle in southern Brazil, p.154-157. In: Riet-Correa F., Pfister J., Schild A.L. \& Wierenga T. (Eds), Poisoning by Plants, Micotoxins and related Toxins. Cab International, Wallingford.

Grecco F.B., Estima-Silva P., Marcolongo-Pereira C., Soares M.P., Collares G. \& Schild A.L. 2011b. Seneciose crônica em ovinos no sul do Rio Grande do Sul. Pesq. Vet. Bras. 31(4):326-330.

Habermehl G.G., Martz W., Tokarnia C.H., Döbereiner J. \& Méndez M.C. 1988. Livestock poisoning in South America by species of the Senecio plant. Toxicon 26(3):275-286.

Harper J.L. \& Wood W.A. 1957. Biological flora of the British Isles: Senecio jacobaea L. J. Ecology 45:617-637.

Hirschmann G.S., Ferro E.A., Franco L., Recalde L. \& Theoduloz C. 1987. Pyrrolizidine alkaloids from Senecio brasiliensis populations. J. Natural Products 50(4):770-772.

Hooper P.T. 1972. Spongy degeneration in the brain in relation to hepatic disease and ammonia toxicity in domestic animals. Vet. Rec. 90:37-38.

Ilha M.R., Loretti A.P., Barros S.S. \& Barros C.S.L. 2001. Intoxicação espontânea por Senecio brasiliensis (Asteraceae) em ovinos no Rio Grande do Sul. Pesq. Vet. Bras. 21:123-138.

Karam F.S.C., Méndez M.C., Jarenkow J.A. \& Riet-Correa F. 2002. Fenologia de quatro espécies tóxicas de Senecio (Asteraceae) na região Sul do Rio Grande do Sul. Pesq. Vet. Bras. 22(1):33-39.
Karam F.S.C., Soares M.P., Haraguchi M., Riet-Correa F., Méndez M.C. \& Jarenkow J.A. 2004. Aspectos epidemiológicos da seneciose na região sul do Rio Grande do Sul. Pesq. Vet. Bras. 24(4):191-198.

Karam F.S.C. \& Jarenkow J.A. 2011. Phenology of Senecio spp. and vegetation cover in the state of Rio Grande do Sul, Brazil, p.158-162. In: Riet-Correa F., Pfister J., Schild A.L. \& Wierenga T. (Eds), Poisoning by Plants, Mycotoxins and related Toxins. CAB International, Wallingford, UK.

Karam F.S.C. \& Motta A. 2011. Pyrrolizidine alkaloids poisoning in cattle in the state of Rio Grande do Sul, Brazil, p.175-178. In: Riet-Correa F., Pfister J., Schild A.L. \& Wierenga T. (Eds), Poisoning by Plants, Mycotoxins and related Toxins. CAB International, Wallingford, UK.

Karam F.S.C., Haraguchi M. \& Gardner D. 2011. Seasonal variation in pyrrolizidine alkaloid concentration and plant development in Senecio madagascariensis Poir. (Asteraceae) in Brazil, p.179-185. In: Riet-Correa F., Pfister J., Schild A.L. \& Wierenga T. (Eds), Poisoning by Plants, Mycotoxins and related Toxins. CAB International, Wallingford, UK.

Kingsbury J.M. 1964. Poisonous Plants of the United States and Canada. Prentice-Hall, Englewood Cliffs, New Jersey. 626p.

Kluwe C.S. 2008. Comunicação pessoal (Condomíno Rural Rossell e Romero, Bagé, RS).

Lucena R.B., Pierezan F., Kommers G.D., Irigoyen L.F., Fighera R.A. \& Barros C.S.L . 2010a. Doenças de bovinos no Sul do Brasil: 6.706 casos. Pesq. Vet. Bras. 30(5):428-434.

Lucena R.B., Rissi D.R., Maia L.A., Flores M.M., Dantas A.F.M., Nobre V.M.T., Riet-Correa F. \& Barros C.S.L. 2010b. Intoxicação por alcalóides pirrolizidínicos em ruminantes e equinos no Brasil. Pesq. Vet. Bras. 30(5): 447-452.

Macedo W. 1984. Levantamento de reconhecimento dos solos do Município de Bagé. Documentos 1, Embrapa/UEPAE, Bagé, RS. 69p.

Madanes N., Vicari R. \& Bonaventura S.M. 1996. Fenologia de las especies de los bordes de caminos en agroecosistemas y su relación con los parámetros climáticos. Parodiana 9(1/2):149-158.

MAF 2010. Senecio within the Asteraceae (daisy/thistle) family. MAF Information Services, Pastoral House, Wellington, New Zealand <http:// www.maf.govt.nz/maf net/rural-nz/sustainable-resource-use/landmanagement/emerging-weeds>

Maia F.C., Medeiros R.B., Pillar V.P., Chollet D.M.S. \& Olmedo M.O.M. 2003. Composição, riqueza e padrão de variação do banco de sementes do solo em função da vegetação de um ecossistema de pastagem natural. Iheringia, Sér. Bot, 58:61-80.

Maia F.C., Medeiros R.B., Pillar V.P. \& Focht T. 2004. Soil seed bank variation patterns according to environmental factors in a natural grassland. Revta Bras. Sementes 26(2):126-137.

MacLachlan N.J. \& Cullen J.M. 1998. Fígado, sistema biliar, e pâncreas exócrino, p.95-131. In: Carlton W.W. \& McGavin M.D. (Eds), Patologia Veterinária Especial de Thomson. $2^{\underline{a}}$ ed. Artes Médicas, Porto Alegre.

McEvoy P., Cox C. \& Coombs E. 1991. Successful biological control of ragwort, Senecio jacobaea, by introduced insects in Oregon. Ecological Applications 1(4):430-442.

Matzenbacher N.I. 1998. O complexo "Senecionoide" (AsteraceaeSenecioneae) no Rio Grande do Sul, Brasil. Tese de Doutorado em Botânica, Instituto de Biociências, UFRGS, Porto Alegre. 274p.

Matzenbacher N.I. \& Schneider A.A. 2008. Nota sobre a presença de uma espécie adventícia de Senecio (Asteraceae) no Rio Grande do Sul, Brasil. Revta Bras. Biociênc. 6:111-115.

Matzenbacher N.I. 2009. Uma nova espécie do gênero Senecio L. (Asteraceae - Senecioneae) no Rio Grande do Sul, Brasil. Iheringia, Sér. Bot, 64(1): 109-113.

Maxie M.G. 2007. Jubb, Kennedy and Palmer's. Pathology of Domestic Animals. Vol.2. $5^{\text {th }}$ ed. Saunders Elsevier, London. 771p.

McClements I., Courtney A.D. \& Malone F.E. 1998. Management and edaphic factors related with the incidence of marsh ragwort, p.40-44. In: Garland T. \& Barr A.C. (Eds), Toxic Plants and other Natural Toxicants. Biddles Ltd, Guildford and King's Lynn, Oxon.

Mendes M.M., Leite M.L., Corrêa G.H. \& Milléo J. 2005. Entomofauna associa-da a Senecio brasiliensis Less. (Asteraceae), e Phaedon confinis (Insecta; 
Coleoptera; Chrysomelidae) como possível agente controlador desta planta tóxica. Publ. UEPG, Ciências Exatas e da Terra, Ciências Agrárias e Engenharias 11:45-53.

Méndez M.C. 1993. Intoxicação por Senecio spp., p.43-57. In: Riet-Correa F., Méndez M.C. \& Schild A.L. (Eds), Intoxicações por Plantas e Micotoxicoses em Animais Domésticos. Editorial Agropecuaria Hemisferio Sur, Montevideo.

Méndez M.C. \& Riet-Correa F. 1993. Intoxication by Senecio tweediei in cattle in southern Brazil. Vet. Human. Toxicol. 35(1):55.

Méndez M.C. \& Riet-Correa F. 2008. Plantas Tóxicas e Micotoxicoses. 2ª ed. Editora e Gráfica Universitária, Pelotas. 298p.

Méndez M.C., Riet-Correa F., Schild A.L. \& Martz W. 1990. Intoxicação experimental por cinco espécies de Senecio em bovinos e aves. Pesq. Vet. Bras. 10(3/4):63-69.

Milléo J., Corrêa G.H., Leite M.L. \& Pedrosa-Macedo J.H. 2006. Comportamento e ciclo de vida de Phaedon confinis (Coleoptera, Chrysomelidae) em condições de laboratório. Revta Bras. Entomol. 50(3):419-422.

Motidome M. \& Ferreira P.C. 1966. Alcalóides de Senecio brasileinsis Less. Revta Fac. Farm. Bioquím., São Paulo, 4(1):13-44.

Osweiler G.D. 1998. Toxicologia Veterinária. Artes Médicas, Porto Alegre. 526 .

Overbeck G.E., Müller S.C., Fidelis A., Pfadenhauer J., Pillar V.D., Blanco C., Boldrini I.I., Both R. \& Forneck E.D. 2007. Brazil's neglected biome: The South Brazilian campos. Perspectives in Plant Ecology Evolution and Systematics 9:101-116.

Pedroso P.M.O., Pescador C.A., Oliveira E.C., Sonne L., Bandarra P.M., Raymundo D.L. \& Driemeier D. 2007. Intoxicações naturais por plantas em ruminantes diagnosticadas no Setor de Patologia Veterinária. Acta Scient. Vet. 35(2):213-218.

Peterson J.E. \& Culvenor C.C.J. 1983. Hepatotoxic pyrrolizidine alkaloids, p.637-671. In: Keeler R.F. (Ed.), Plant and Fungal Toxins: Handbook of natural toxins. Vol.1. Marcel Dekker, New York.

Radostits O.M., Gay C.C., Blood D.C. \& Hinchcliff K.W. 2002. Veterinary Medicine. $10^{\text {th }}$ ed. W.B. Saunders, London. 1881p.

Riet-Correa F. \& Méndez M.C. 2007. Intoxicações por Plantas e Micotoxinas, p.99-219. In: Riet-Correa F., Schild A.L., Lemos R.A.A. \& Borges J.R.J. (Eds), Doenças de Ruminantes e Eqüídeos. Vol.2. Editora Pallotti, Santa Maria, RS.

Rissi D.R., Rech R.R., Pierezan F., Gabriel A.L., Trost M.E., Brum J.S., Kommers G.C. \& Barros C.S.L. 2007. Intoxicações por plantas e micotoxinas associ- adas a plantas em bovinos no Rio Grande do Sul: 461 casos. Pesq. Vet. Bras. 27:261-268.

Rissi D.R., Pierezan F., Oliveira-Filho J.C., Lucena R.B., Carmo P.M.S. \& Barros C.S.L. 2010. Abordagem diagnóstica das principais doenças do sistema nervoso de ruminantes e equinos no Brasil. Pesq. Vet. Bras. 30(11): 958-967.

Romero N.F. 1998. Manejo Fisiológico dos Pastos Nativos Melhorados. Livraria e Editora Agropecuária Ltda, Guaíba, RS. 110p.

Saint-Hilaire A. de. 1887. Voyage à Rio Grande do Sul, Brésil (Tradução de Leonam de Azeredo Penna, 1974. Viagem ao Rio Grande do Sul). Itatiaia, Belo Horizonte. 215p.

Sandini T.M., Udo M.S.B. \& Spinosa H.S. 2010. Interferência no desenvolvimento físico e neurocomportamental da prole de ratas causada pela exposição ao extrato de Senecio brasiliensis durante o período pré-natal. Anais 4ª Semana Científica Benjamin Eurico Malucelli, Departamento de Análises Clínicas e Toxicológicas, Universidade de São Paulo, p.35-36. (Resumo)

Silva A.A. \& Silva J.F. 2009. Tópicos em Manejo de Plantas Daninhas. Editora UFV, Universidade Federal de Viçosa. 367p.

Soares M.P., Riet-Correa F., Méndez M.C., Rosa F.G. \& Carreira E.G. 2000. Controle biológico de Senecio spp. com pastoreio de ovinos. Anais II Reunión Argentina de Patologia Veterinaria, Faculdad de Ciencias Veterinarias, Universidad Nacional del Nordeste, Corrientes, Argentina, p.79. (Resumo)

Solera M., Hefler S.M. \& de Paula M.C.Z. 2007. Estudo das interações entre insetos e Senecio brasiliensis Less. (Asteraceae) em área experimental no campus da Pontifícia Universidade Católica do Paraná, Toledo, Brasil. Estud. Biol. 29(66):81-87.

Spinosa H.S., Górniak S.L. \& Palermo-Neto J. 2008. Toxicologia Aplicada à Medicina Veterinária. Editora Manole, São Paulo. 942p.

Summers B.A., Cummings J.F. \& deLahunta A. 1995. Degenerative diseases of the central nervous system, p.208-350. In: Summers B.A., Cummings J.F. \& de Lahunta A. (Eds), Veterinary Neuropathology. Mosby, St Louis.

Thompson K., Grime J.P. \& Mason G. 1977. Seed germination in response to diurnal fluctuations of temperature. Nature 267:147-149.

Tokarnia C.H. \& Döbereiner J. 1984. Intoxicação experimental por Senecio brasiliensis (Compositae) em bovinos. Pesq. Vet. Bras. 4(2):39-65.

Tokarnia C.H., Döbereiner J. \& Peixoto P.V. 2000. Plantas hepatotóxicas, 80110. In: Ibid. (Eds), Plantas Tóxicas do Brasil. Editora Helianthus, Rio de Janeiro. 310p.

Villalba J. \& Fernández G. 2005. Otra flor amarilla peligrosa: Senecio madagascariensis. Tambo 150:46-48. 